\title{
Corela
}

Cognition, représentation, langage

HS-17 | 2015

Regards sur l'interdisciplinarité en science du langage

\section{Les discours des manuels de philosophie en France et au Brésil : une analyse contrastive}

Daniela Nienkötter Sardá

\section{OpenEdition}

\section{Journals}

Édition électronique

URL : http://journals.openedition.org/corela/3706

DOI : $10.4000 /$ corela.3706

ISSN : $1638-573 \mathrm{X}$

Éditeur

Cercle linguistique du Centre et de l'Ouest - CerLICO

Référence électronique

Daniela Nienkötter Sardá, « Les discours des manuels de philosophie en France et au Brésil : une analyse contrastive », Corela [En ligne], HS-17 | 2015, mis en ligne le 30 mai 2015, consulté le 19 avril 2019. URL : http://journals.openedition.org/corela/3706 ; DOI : 10.4000/corela.3706

Ce document a été généré automatiquement le 19 avril 2019

\section{(c) (i) (2)(2)}

Corela - cognition, représentation, langage est mis à disposition selon les termes de la licence Creative Commons Attribution - Pas d'Utilisation Commerciale - Partage dans les Mêmes Conditions 4.0 International. 


\title{
Les discours des manuels de philosophie en France et au Brésil : une analyse contrastive
}

\author{
Daniela Nienkötter Sardá
}

\section{Introduction}

1 L'enseignement de la philosophie est l'objet d'une discussion récente en France, car on envisage la possibilité de l'enseigner avant la classe de terminale, dès la seconde. Au Brésil, la philosophie est récemment (re-)devenue une matière scolaire obligatoire dans les lycées.

2 En lisant des manuels scolaires de philosophie français et brésiliens, nous avons eu l'impression qu'il y avait des différences entre leurs discours. Partant de cette impression et sachant que ces manuels sont rédigés d'après des programmes officiels, nous pensons que l'enseignement de la philosophie doit avoir des buts différents dans les deux pays. Il s'agit donc pour nous de chercher des représentations de la philosophie susceptibles d'être dégagées d'un corpus choisi de manuels scolaires de philosophie français et brésiliens.

Dans cet article, nous voulons exposer notre recherche en analyse du discours, en montrant en quoi une approche interdisciplinaire - celle de la linguistique de discours comparative - se révèle essentielle pour une comparaison des discours de manuels scolaires de philosophie.

4 Ainsi, nous exposerons d'abord le contexte de l'enseignement de la philosophie en France et au Brésil (partie 1). Par la suite, nous présenterons des questions relatives au corpus (partie 2) ainsi qu'au modèle interdisciplinaire adopté pour son analyse (partie 3). Des résultats provisoires seront présentés (partie 4) avant de proposer, en guise de conclusion, quelques hypothèses et pistes de réflexion sur les représentations de la philosophie qui circulent dans les communautés ethnolinguistiques française et brésilienne. 


\section{Le contexte de l'enseignement de la philosophie dans les lycées français et brésiliens}

5 Si nous avons eu l'impression de l'existence de différences lors d'une première lecture des manuels de philosophie français et brésiliens, nous constatons, après une analyse plus minutieuse du système d'enseignement des deux pays, qu'il y a plus de similitudes dans le mode d'organisation de cet enseignement en France et au Brésil que ce que l'on pouvait en supposer dans un premier temps. Dans les deux pays, l'enseignement de la philosophie est obligatoire dans les lycées, s'effectue dans le cadre d'une éducation centralisée et est abordé de manière thématique.

6 L'enseignement obligatoire de la philosophie dans les lycées français et brésiliens présente néanmoins des histoires différentes. Si en France la philosophie est une discipline obligatoire dans l'enseignement secondaire - dans la dernière année du lycée ${ }^{1}$ depuis le Premier Empire, avec une éclipse de 1852 à 1863 (cf. Sherringham, 2006 : 61), cela n'est pas le cas au Brésil, où l'enseignement de la philosophie, actuellement présent dans les trois années de lycée, est caractérisé par une histoire plus marquée d'allées et venues.

7 L'enseignement de la philosophie, avec celui de la sociologie, est devenu obligatoire au Brésil, dans tous les lycées, publics et privés, depuis le 02 juin 2008, avec la promulgation de la loi fédérale $\mathrm{n}^{\mathrm{o}} 11.684^{2}$. L'implémentation de cet enseignement obligatoire est l'objet d'une lutte qui remonte à l'époque post-dictature militaire, puisque la philosophie a été supprimée des curricula scolaires en 1971, lorsque la loi $n^{\circ}$ 5.692/71 a interdit son enseignement dans le pays. Avant cette interruption, l'enseignement de la philosophie était obligatoire depuis 1942, dans le gouvernement de Getúlio Vargas, et avant encore : d'autres interruptions ont eu lieu, mais cet enseignement est présent dans les programmes « [...] depuis la création de la première école d'enseignement secondaire de la compagnie de Jésus à Salvador de Bahia en 1553 » (Kohan, 2007 : 78). L'enseignement actuellement obligatoire de la philosophie au Brésil vise la formation à la citoyenneté, le développement intellectuel et de la pensée critique (Brasil - Secretaria de Educação Média e Tecnológica, $2000: 44$ ).

En France, l'enseignement de la philosophie dans les lycées est lié à «[...] la formation du “citoyen éclairé" [...] 》 (Sherringham, $2006: 61$ ). Comme au Brésil, cet enseignement n'est pas confondu avec celui de la religion, de la morale ou de l'éthique. D'après le rapport de l'UNESCO sur l'enseignement de la philosophie en Europe et Amérique du Nord,

[...] les principales caractéristiques nationales qui déterminent les modes d'organisation de l'enseignement de la philosophie au niveau secondaire sont les suivantes : l'organisation centralisé ou décentralisée du système d'éducation au niveau national, les liens historiques plus ou moins forts entre cours de philosophie et cours de religion et de morale, la conception de l'apport spécifique de la philosophie à la formation scientifique générale des jeunes (c'est cette conception qui détermine le statut optionnel ou obligatoire de la philosophie selon les sections de spécialisation), les conceptions de pédagogie, etc. (UNEsco, $2011: 41$ ).

On observe également qu'en France, autant qu'au Brésil, l'enseignement de la philosophie fait partie d'une éducation centralisée. Cet aspect est important pour notre recherche, puisque nous cherchons des représentations de la philosophie susceptibles d'être dégagées des discours des manuels français et brésiliens. Ce serait difficile de comparer 
les manuels d'un pays dans lequel l'enseignement est centralisé aux ouvrages d'un pays dans lequel chaque région décide de façon autonome des programmes scolaires.

Mais l'enseignement centralisé de la philosophie en France et au Brésil présente, à notre avis, une particularité dans chaque pays étudié. En France, ayant terminé leurs études de Terminale, tous les lycéens passent l'épreuve de philosophie du baccalauréat au même jour et à la même heure, en répondant aux mêmes questions ${ }^{3}$. Au Brésil, dans le cadre du Programme National du Livre Didactique, le PNLD, tout élève d'école publique, du Nord au Sud, reçoit gratuitement un exemplaire du livre de philosophie choisi par le professeur de son lycée (parmi les trois options qui constituent le guide du livre didactique de philosophie). Le PNLD existe depuis 1996, mais le guide de manuels de philosophie est paru en 2011, étant donné le caractère obligatoire récent de cette discipline.

Les livres distribués par le PNLD au Brésil, ainsi que les manuels français, sont organisés par thèmes, ce qui montre que la France et le Brésil suivent tous les deux une approche thématique, par opposition à l'approche historique (cf. UNESCO, 2011 : 50). Cette approche présente quelques avantages. En France, l'un des avantages de ce système, d'après Sherringham (apud UNEsco, $2011: 51$ ), c'est que le cours de philosophie «[...] ne se décline jamais comme un exposé chronologique de doctrines mortes ou (dé)passées ». Au Brésil, l'approche thématique offre une liberté plus large au professeur ${ }^{4}$, qui peut choisir, à chaque étape de son enseignement, de nouveaux thèmes à être travaillés en fonction de ses besoins tout comme ceux de ses élèves.

Enfin, il est important de noter qu'en France la philosophie est enseignée en Terminale dans les voies générales et technologiques. Dans la voie générale, il s'agit de $8 \mathrm{~h}$ en série littéraire, $4 \mathrm{~h}$ en série économique-sociale et $2 \mathrm{~h}+1 \mathrm{~h}$ en série scientifique, tandis que dans la voie technologique, il s'agit de $1 \mathrm{~h}+1 \mathrm{~h}$. (cf. Sherringham, $2006: 61$ ). Au Brésil, la philosophie est enseignée deux heures par semaine au moins ${ }^{5}$, pendant les trois années du lycée.

\section{Le corpus}

Le corpus de manuels scolaires de philosophie français et brésiliens est résolument contemporain. Nous avons choisi, pour commencer, six manuels de philosophie, car nous envisageons une "analyse fine » des discours de ces manuels, c'est-à-dire une analyse détaillée et diversifiée (il s'agit de mobiliser plusieurs catégories d'analyse), partant noninformatisée.

Étant donné la diversité des manuels de philosophie disponibles en France, ils ont été choisis parmi les plus vendus, à savoir :

- Delattre, michel (dir.) (2012), Philosophie. Terminales L, ES, S. Paris : Hatier (dorénavant [Philosophie Terminales]);

- HANSEN-LøVE, Laurence (dir.) (2004), Philosophie. Anthologie Terminales L. ES. S., Paris : Belin (dorénavant [Philosophie Anthologie]) ;

- RUSS, Jacqueline \& LEGUIL, Clotilde (2012), Les chemins de la pensée. Philosophie Terminales L - ES $-S$, Paris : Bordas (dorénavant [Chemins]).

Pour les manuels brésiliens, le choix s'est avéré plus simple car il n'y a que trois manuels qui figurent dans le guide du livre didactique de philosophie, à savoir :

- CHAUI, Marilena (2011), Iniciação à Filosofia, São Paulo : Ática (dorénavant [Iniciação]) ; 
- cotrim, Gilberto \& FERnAndes, Mirna (2010), Fundamentos de filosofia, São Paulo : Saraiva (dorénavant [Fundamentos]);

- MARTins, Maria Helena Pires \& ARANHA, Maria Lúcia de Arruda (2009), Filosofando : introdução à filosofia, São Paulo : Moderna (dorénavant [Filosofando]).

S'agissant d'une analyse fine des discours, notre analyse débute par les manuels supra. Tout d'abord, on effectue une sous-division de ce matériel dans des sous-corpus de travail ${ }^{6}$, puis au fur et à mesure de la recherche, on peut, éventuellement, ajouter d'autres manuels à ce corpus, afin de garantir la représentativité des résultats de recherche. Par représentativité, nous entendons le fait que les résultats de l'analyse sont généralisables. Puisque nous faisons une comparaison, il est important que les résultats de l'analyse soient représentatifs des discours des manuels de philosophie de chaque pays étudié.

\section{Un modèle interdisciplinaire pour l'analyse des discours de manuels de philosophie français et brésiliens}

Pour l'analyse de notre corpus, nous adoptons le modèle de description et interprétation conçu par von Münchow (2004) dans le cadre de la linguistique de discours comparative ( cf. Figure 1).

Figure 1 : Modèle de description et interprétation

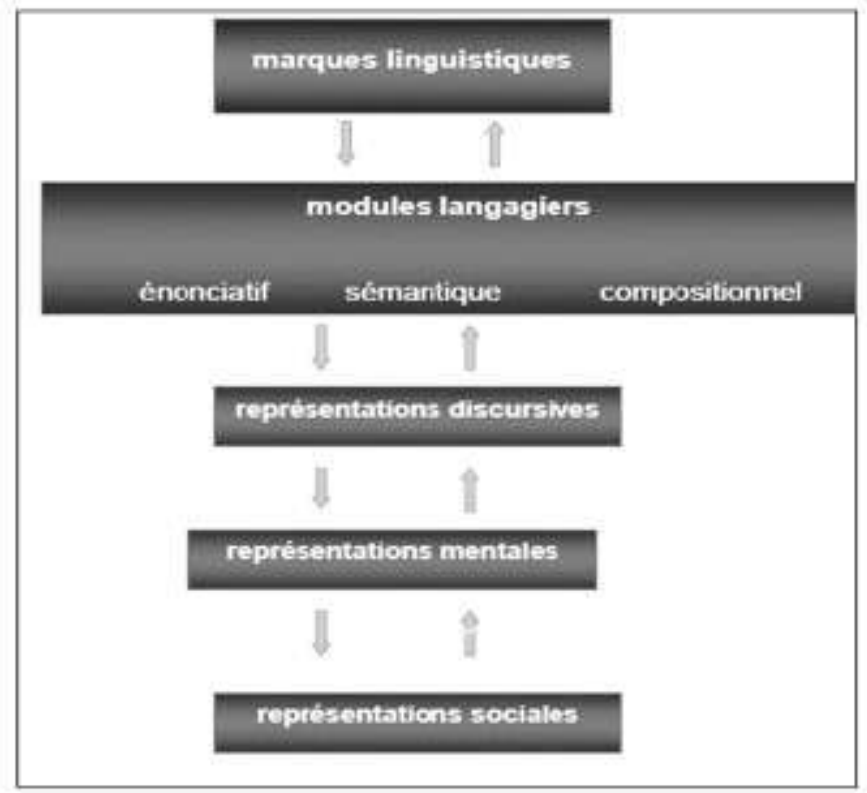

D'après von Münchow (2004 : 49), la «[...] linguistique de discours comparative a comme objet la comparaison descriptive et interprétative de genres discursifs d'au moins deux communautés ethnolinguistiques». Dans notre cas, nous comparerons des documents issus de deux communautés, la France et le Brésil. Le genre discursif en question est le genre manuel scolaire de philosophie.

Le modèle de description et d'interprétation ( $c f$. Figure 1 infra) implique une approche interdisciplinaire à plusieurs niveaux. Dans notre cas, on mobilisera, bien entendu, la 
linguistique et la philosophie. En outre, la psychologie sociale entre dans notre recherche par le biais du concept de représentation sociale, qui est intrinsèque au modèle adopté.

Tout d'abord, la recherche commence par la linguistique. C'est cette discipline qui fournira des catégories descriptives pour l'analyse de notre corpus. Nous analyserons le corpus français et le corpus brésilien en observant plusieurs phénomènes linguistiques, qui vont de l'usage des déictiques à celui du discours rapporté dans les manuels, et dont les emplois différent d'une langue à l'autre.

Une fois accomplie cette analyse linguistique, on part à la recherche de représentations discursives, mentales, sociales. On considère que «[...] [1]es représentations mentales de l'auteur [des manuels de philosophie, dans notre cas] sont influencées par les représentations sociales (cf. Guimelli 1999 : 63) en cours dans la communauté en question, tout en pouvant se construire contre ces représentations sociales » (von Münchow, 2011 : 13-14).

Le concept de représentation sociale naît, en psychologie sociale, de la reformulation qu'a faite Serge Moscovici (1961) du concept de représentation collective de Durkheim (1898) :

L'«ordre de phénomènes" appelé représentations collectives a été étudié initialement par le sociologue Durkheim et a joué un rôle très important dans son œuvre (Lukes 1973). Moscovici (1976), en tant que psychologue social, a traité du même ordre de phénomènes dans son travail sur les représentations sociales de la psychanalyse mais d'une manière différente de celle du fondateur de la sociologie moderne. (Doise \& Palmonari, 1997 : 15).

En psychologie sociale on peut définir le concept de représentation sociale « [...] comme un ensemble de connaissances de sens commun à visée pratique relatives à un objet social. La représentation est spécifique à un groupe social donné, elle permet la prise en compte d'une réalité commune » (Jodelet, 1989 apud Ionescu \& Blanchet, 2008 : 19). Nous chercherons cette " pensée commune » - les représentations - qui contribuent à façonner (tout en étant façonnées par) les discours des manuels scolaires de philosophie français et brésiliens.

Finalement, en suivant le modèle de description et interprétation, c'est-à-dire en partant des observations linguistiques pour aller vers des interprétations simples sur les représentations qui circulent dans une communauté ethnolinguistique sur un thème donné - dans notre cas, sur la philosophie -, nous devrons encore relier ces représentations à des causalités, conformément à ce qu'explique von Münchow $(2011: 14)$ : "Après l'interprétation "simple", les représentations peuvent être reliées - par l'interprétation de l'analyste, là encore - à des causalités institutionnelles, historiques, matérielles, etc. (interprétation "causale") ».

\section{Premiers résultats}

Afin de rendre plus clair ce qui a été exposé supra, nous présenterons une première analyse du corpus français et brésilien.

\subsection{La philosophie dans les manuels français}

Les manuels français ne consacrent pas une unité ou un chapitre pour expliquer à leurs lecteurs ce qu'est la philosophie, contrairement à ce que font les auteurs des manuels brésiliens ( $c f$. partie 4.2). Nous avons été amenées à faire une analyse des choix 
thématiques dans les manuels français, afin de rechercher si on ne parlait pas de la philosophie à d'autres moments dans ces ouvrages. Cette analyse des choix thématiques concerne le module sémantique du modèle de description et interprétation (cf. partie 3).

D'abord, on observe que les trois ouvrages français de notre corpus présentent tous, avant le sommaire, un résumé du programme de philosophie pour les classes terminales des séries littéraire, économique-sociale et scientifique (programme du ministère de l'Éducation nationale du 19 juin 2003). Les cinq notions présentes dans le programme sont le sujet, la culture, la raison et le réel, la politique et la morale. Ces notions sont ensuite divisées en sous-notions. Les auteurs présentent aussi la liste de repères et d'auteurs qui doivent être travaillés en Terminale selon ce même programme. Parmi ces sous-notions et repères, il n'est jamais question de la philosophie en tant que telle. Le programme étant suivi par les auteurs, on ne trouvera pas « la philosophie » comme notion ou repère abordé dans les manuels français.

Cependant, il est question dans les manuels français de l'exposition de quelques textes où les philosophes eux-mêmes parlent de la philosophie. Cette thématique apparaît dans [Philosophie Anthologie] aux pages 256 (Kant. Mathématique et philosophie) et 295 (Hegel. La vérité en philosophie), et dans [Chemins] aux pages 76 (Socrate et la philosophie), 198 (Descartes. Qu'est-ce que la philosophie ?) et 308 (Hegel. La philosophie est un système et un cercle).

Dans la présentation d'un extrait de Critique de la raison pure ${ }^{7}$, les auteurs de [Philosophie Anthologie] nous apprennent que :

(1) La démarche philosophique ne peut être assimilée à la démarche mathématique, parce qu'elle appréhende ses objets à travers les seuls concepts, sans rapport immédiat à une intuition qui correspondrait à ces concepts. La démonstration que la chose même se montre. [Philosophie Anthologie], p. 256.

De même, on apprend qu'une autre conception de la vérité de la philosophie est formulée par Hegel dans Leçons sur l'histoire de la philosophie. Introduction du cours de Berlin (1820) ${ }^{8}$ :

(2) Quelle vérité peut-on attendre de la philosophie ? Il est banal de souligner combien les philosophes sont en désaccord : si l'on admet, pour des raisons subjectives, qu'une seule philosophie dit vrai, les autres apparaîtront nécessairement fausses. En examinant le cheminement de la vérité à travers les différents systèmes, Hegel aboutit ici à une tout autre conception. [Philosophie Anthologie], p. 295.

31 Les auteures de [Chemins] choisissent comme exemple un texte de Hegel extrait du tome 1 de Logique et, par l'emploi du déictique vous, conseillent aux lecteurs de le faire dialoguer avec des textes de Nietzsche et Kierkegaard:

(3) Dans ce texte, Hegel explicite l'idée que seule la philosophie rigoureuse forme un système, c'est-à-dire une unité organique d'éléments interdépendants, un ensemble cohérent. Toutes les philosophies n'ont pourtant pas pris la forme systématique. Songez ${ }^{10}$, par exemple, aux réflexions de Nietzsche ou de Kierkegaard (voir Kierkegaard, texte 2). [Chemins], p. 308-309.

Après chacune de ces présentations exemplifiées par (1), (2) et (3), suit le texte du philosophe en question. Nous voyons, alors, que la philosophie parle d'elle-même : il n'est pas vraiment question d'expliquer, dans les manuels français, ce qu'elle est. Il faudra, bien sûr, que nous analysions plus à fond le corpus à l'aide d'autres catégories d'analyse, mais il nous semble déjà que, dans la communauté française, la philosophie est représentée comme une discipline qui n'a pas besoin de se justifier, c'est-à-dire d'exposer sa raison 
d'être. Il n'est pas non plus question de justifier son utilité, comme ce sera le cas dans les manuels brésiliens ( $c f$. partie 4.2 infra).

\subsection{La philosophie dans les manuels brésiliens}

Contrairement aux manuels français, les manuels brésiliens consacrent une bonne partie de leurs pages à expliciter ce qu'est la philosophie. La première unité de [Iniciação] est intitulée A filosofia (La philosophie) et va des pages 06 à 67. [Fundamentos] consacre presque quatre-vingts pages à l'explication de ce qu'est la philosophie (pp. 10-88), dans une unité intitulée Introdução à filosofia (Introduction à la philosophie). Finalement, [Filosofando] dédie les pages 12 à 43 à ce sujet, dans une unité intitulée Descobrindo a Filosofia (Découvrir la Philosophie).

La lecture de ce sous-corpus nous montre qu'au Brésil il y a une vision assez répandue de la philosophie comme une discipline abstraite, difficile, voire inutile, comme on le voit dans l'exemple (4):

(4) A filosofia é um ramo do conhecimento que tem a fama de ser bastante abstrato e de difícil compreensão para grande parte das pessoas. [Fundamentos] p. 09.

[La philosophie est une branche de la connaissance qui a la réputation d'être très abstraite et de compréhension difficile pour la majorité des gens.]

Nous voyons dans cet exemple que l'idée selon laquelle la philosophie est très abstraite et de compréhension difficile serait déjà un fait social au Brésil, d'après les auteurs, qui utilisent les termes « réputation » et « la majorité des gens ».

Dans l'exemple (5), extrait du manuel [Iniciação], c'est avec un passage en discours direct que l'auteure "simule » la question que, selon elle, de " nombreuses " personnes se posent au sujet de la philosophie au Brésil, à savoir, celle de son utilité immédiate :

(5) Ora, muitos fazem uma outra pergunta: “Afinal, para que filosofia?" É uma pergunta interessante. Não vemos nem ouvimos ninguém perguntar, por exemplo, "Para que matemática ou física?" [...] Mas todo mundo acha natural perguntar: "Para que filosofia?" [...] Essa pergunta, "Para que filosofia?", tem a sua razão de ser. Em nossa cultura e em nossa sociedade, costumamos considerar que alguma coisa só tem o direito de existir se tiver alguma finalidade prática muito visível e de utilidade imediata. [...] [Iniciação] p. 20.

[Or, nombreux sont ceux qui posent une autre question: « Finalement, pourquoi [à quoi sert] la philosophie ? "C'est une question intéressante. Nous ne voyons ni écoutons personne demander par exemple, "Pourquoi les mathématiques ou la physique ? " [...] Mais tout le monde trouve naturel de demander : « Pourquoi la philosophie ? » [...] Cette question, « Pourquoi la philosophie ? ", a sa raison d'être. Dans notre culture et dans notre société, nous avons l'habitude de considérer que quelque chose a le droit d'exister seulement si cela a une finalité pratique très visible et d'utilité immédiate [...]]

Or, si les membres d'une communauté ethnolinguistique se demandent quelle est l'utilité d'une telle discipline, on suppose qu'il y circule une représentation de cette discipline comme inutile. Cette hypothèse est confirmée par le discours des manuels brésiliens, puisque les auteurs de [Iniciação] et de [Filosofando], dans les exemples (5) supra et (6) infra, se positionnent contre cette représentation. Ainsi, l'auteure de [Iniciação], dans (5), se demande pourquoi nous n'entendons " personne demander, par exemple, "Pourquoi les mathématiques ou la physique?" » et conclut en affirmant, avec l'emploi d'un nous inclusif, que "dans notre culture et dans notre société »- à savoir la culture et la société brésilienne à laquelle l'auteure appartient - « nous avons l'habitude de considérer que 
quelque chose a le droit d'exister seulement si cela a une finalité pratique très visible et d'utilité immédiate [...] ».

Les auteurs de [Filosofando], dans l'exemple (6) infra, se positionnent également contre la représentation de la philosophie comme inutile. Ils emploient les mots " utilité » et « inutilité » entre guillemets, justement pour créer un effet de mise à distance : pour eux, il n'y a aucun sens à envisager la philosophie sous l'angle de l'utilité ou de l'inutilité. En somme, nous observons que la représentation de la philosophie comme inutile est une représentation extérieure à la communauté des auteurs des manuels de cette discipline au Brésil. L'emploi du syntagme "cette ligne de pensée " associé à ceux qui voient la philosophie comme inutile ne fait que renforcer cette interprétation.

(6) Afinal, qual é a "utilidade" da filosofia? Vivemos num mundo que valoriza as aplicações imediatistas do conhecimento. O senso comum aplaude a pesquisa científica que visa à cura do câncer ou da aids; a matemática no ensino médio seria importante por "cair" no vestibular; a formação técnica do advogado, do engenheiro, do fisioterapeuta prepara para o exercício dessas profissões. Diante disso, não é raro que alguém indague: Para que estudar filosofia se não vou precisar dela na minha vida profissional? De acordo com essa linha de pensamento, a filosofia seria realmente "inútil", já que não serve para qualquer alteração imediata de ordem prática. No entanto, a filosofia é necessária. Por meio daquele "olhar diferente", ela busca outra dimensão da realidade além das necessidades imediatas nas quais o indivíduo encontra-se mergulhado: ao tornar-se capaz de superar a situação dada e repensar o pensamento e as ações que ele desencadeia, o indivíduo abre-se para a mudança. Tal como o artista, a que nos referimos na abertura do capítulo, ao filósofo incomoda o imobilismo das coisas feitas e muitas vezes ultrapassadas. Por isso mesmo, a filosofia pode ser "perigosa", por exemplo, quando desestabiliza o status quo ao se confrontar com o poder. [Filosofando] p. 16-17.

[Enfin, quelle est l'« utilité » de la philosophie ? Nous vivons dans un monde qui valorise les applications immédiates de la connaissance. Le sens commun applaudit la recherche scientifique qui vise à la cure du cancer ou du sida ; les [sujets de] mathématiques dans le lycée seraient importants parce qu'ils " tombent » au vestibular ${ }^{11}$; la formation technique d'avocat, d'ingénieur, de kinésithérapeute prépare pour l'exercice de ces professions. Devant cela, il n'est pas rare que quelqu' un demande : Pourquoi étudier la philosophie si je ne vais pas en avoir besoin dans ma vie professionnelle ? En accord avec cette ligne de pensée, la philosophie serait réellement "inutile", puisqu'elle ne sert pas pour une quelconque altération immédiate d'ordre pratique. Cependant, la philosophie est nécessaire. Par le moyen de ce « regard différent ", elle cherche une autre dimension de la réalité au delà des nécessités immédiates dans lesquelles l'individu se trouve plongé : en se rendant capable de surmonter la situation donnée et repenser la pensée et les actions qu' elle déchaîne, l'individu s'ouvre au changement. Tel l'artiste, auquel nous avons fait référence dans l'ouverture du [de ce] chapitre, le philosophe est gêné par l' immobilité des choses déjà faites et très souvent dépassées. C'est pour cela même que la philosophie peut être "dangereuse », par exemple, lorsqu'elle déstabilise le statu quo en se confrontant avec le pouvoir.]

39 Étant donné cette représentation négative de la philosophie au Brésil, les auteurs des manuels, plus particulièrement ceux de [Fundamentos], essayent d'argumenter en faveur de cette discipline, afin de persuader le lecteur, avec tous les moyens rhétoriques possibles, de son utilité, conformément à ce qu'attestent les exemples (7) et (8) infra :

(7) Você provavelmente, em algum momento, já deu os primeiros passos na experiência filosófica e nem se deu conta disso. [Fundamentos] p. 10.

[Probablement tu as déjà fait, à un moment quelconque, les premiers pas dans l' expérience philosophique et tu ne t'en es même pas rendu compte.] 
(8) Certamente você já passou, em algum momento, por uma experiência parecida [de estranhamento e de questionamento], após algum acontecimento marcante em sua vida. Pode ter sido durante uma viagem ao estrangeiro, na morte de um ser querido, em uma grande decepção amorosa, ou em muitas outras circunstâncias distintas. E aí começou a se questionar, mesmo que superficial e brevemente, sobre sua vida e a existência em geral. Pois, então, você estava tendo uma experiência filosófica, ainda que rudimentar. Estava dando os primeiros passos no filosofar. [Fundamentos] p. 12.

[Certainement tu as déjà passé, à un moment donné, par une expérience pareille $[\mathrm{d}$ 'étrangeté ou de questionnement], après un certain événement marquant dans ta vie. Il se peut qu'il ait eu lieu durant un voyage à l'étranger, suite à la mort d'un être cher, lors d'une grande déception amoureuse, ou à l'occasion d'autres nombreuses circonstances différentes. Et alors tu as commencé à te questionner, bien que superficiellement et brièvement, sur la vie et l'existence en général. Car, alors, tu étais en train d'avoir une expérience philosophique, quoique rudimentaire. Tu étais en train de faire les premiers pas dans l'expérience de philosopher.]

L'exemple (8) présente la structure typique d'une concession argumentative : « La première proposition est marquée par des éléments lexicaux relevant de la modalité épistémique. La deuxième est introduite le plus souvent par un mot adversatif (conjonction ou adverbe) »(Morel, 1996 : 18). Ainsi, les auteurs de [Fundamentos] introduisent - et cela depuis les pages précédentes, comme l'atteste l'exemple (7) - des passages qui argumentent en faveur d'une éventuelle expérience philosophique chez les lecteurs, moyennant l'emploi des adverbes « probablement » et « certainement ». Mais ils sont prudents en rappelant, moyennant l'emploi de « mesmo que » (bien que) et " ainda que » (quoique), le caractère " superficiel ", "bref » et " rudimentaire " de ces expériences par rapport à celles des philosophes.

41 Dans les exemples (9) infra, le comparatif « plus » est associé à la construction d'une vie meilleure, qui serait possible avec la philosophie : une vie « plus juste, sage, généreuse et heureuse $»$.

(9) Isso ampliará nossa maneira [de] ver as coisas e a (sic) nós mesmos, ampliando nossos horizontes e possibilidades de escolha para a construção de uma vida mais justa, sábia, generosa e feliz. [Fundamentos] p. 35.

[Ça élargira notre manière [de] voir les choses et nous-mêmes, en étendant nos horizons et possibilités de choix pour la construction d'une vie plus juste, sage, généreuse et heureuse.]

Finalement, nous observons que les auteurs de [Fundamentos] vont jusqu'au bout dans la défense de la philosophie, en recourant à l'affect pour persuader ${ }^{12}$ les lecteurs de la valeur positive de cette discipline. L'exemple (10) en est le témoin. Les auteurs emploient le suffixe « -inha » dans « guardadinhas » (bien gardées), qui, en portugais, signale l'intimité avec l'interlocuteur et finissent avec un « Tu ne vas pas le regretter » :

(10) [...] mas tenha paciência. Tanto em filosofia como na vida em geral, é importante não ser precipitado nem preconceituoso, como recomendou o próprio Descartes, principalmente quando se trata de aprender. E é isso o que você está fazendo agora: aprendendo a aprender, aprendendo a filosofar. Assim, considere, primeiramente, que você ainda tem pouca "experiência" filosófica e conhecimento reduzidos nessa área. Além disso, você é jovem, e a filosofia é algo para toda a vida. Muitos temas ou explicações oferecidos por determinado pensador fazem mais sentido em certas etapas de nossa existência do que em outras. Portanto, vá com calma: se algumas pistas fornecidas por ele não parecem agora ser úteis ou significativas para você, deixe-as guardadinhas em um canto de sua memória até surgir o momento adequado de resgatá-las. Você não vai se arrepender disso. 
[Fundamentos] p. 46.

[[...] mais aie la patience. Dans la philosophie autant que dans la vie en général, il est important de ne pas se précipiter ni d'avoir des préjugés, comme l'a recommandé Descartes lui-même, surtout lorsqu'il s'agit d'apprendre. Et c'est cela que tu es en train de faire maintenant : apprendre à apprendre, apprendre à philosopher. Ainsi, considère, premièrement, que tu as encore peu d'« expérience » philosophique et des connaissances réduites dans ce domaine. De plus, tu es jeune, et la philosophie est quelque chose pour toute la vie. Beaucoup de thèmes ou d'explications données par un penseur quelconque font plus de sens dans certaines étapes de notre existence que dans d'autres. Partant, vas-y doucement : si quelques pistes fournies par lui ne semblent plus être utiles maintenant ou significatives pour toi, conserve-les bien gardées [guardadinhas] dans un coin de ta mémoire jusqu'à ce que surgisse le moment adéquat pour les reprendre. Tu ne vas pas le regretter.]

\section{Conclusion}

43 Cette analyse préliminaire de notre corpus nous a amené à quelques hypothèses sur les représentations qui circulent dans les communautés françaises et brésiliennes à propos de la philosophie. Dans cette communauté-ci, la philosophie doit se justifier, et il nous semble qu'il y circule une représentation de cette discipline comme étant abstraite, difficile et inutile; représentation contestée par les auteurs des manuels. Dans cette communauté-là, en revanche, la philosophie n'a pas besoin d'expliquer ce qu'elle est ni de justifier son utilité supposée.

Or, ce dont nous disposons jusqu'à présent, ce sont des hypothèses sur les représentations de la philosophie en France et au Brésil. Ces représentations peuvent avoir des causes diverses. D'autres disciplines des sciences humaines pourront nous aider dans cette recherche des causes, à savoir :

- l'histoire (de l'enseignement de la philosophie dans les deux pays). La récente (re-)insertion de la philosophie comme matière scolaire au Brésil n'est pas un fait négligeable. Conformément à ce que nous expose Carvalho (2012 : 128), pendant les années de la dictature militaire au Brésil (1964-1985), « [...] toute une génération n'a eu accès à la philosophie que de manière informelle, et son enseignement tout comme son étude régulière étaient donc restreints aux universités [...] ». Aujourd'hui, encore selon l'auteur $(2012: 126)$, «[...] on se trouve face à une scène [...] inverse, où la philosophie s'étend depuis les écoles secondaires (lycées) et tout au long de leurs trois années [...] ». Tout cela étant récent, il est naturel que les manuels brésiliens doivent expliquer d'avantage ce qu'est la philosophie. En France, celle-ci n'a pas besoin d'autant d'explications, puisque, nous l'avons vu (cf. partie 1), la philosophie est une matière scolaire enseignée dans la dernière année du secondaire depuis le Premier Empire presque sans interruption (cf. Sherringham, 2006).

- la didactique et la pédagogie. La philosophie, ainsi que les autres matières scolaires, n'est certes pas enseignée de la même manière dans les deux pays en question. En France, la philosophie est sanctionnée par l'examen du baccalauréat, et des notions précises doivent être travaillées dans son intégralité ${ }^{13}$, en classe, par les enseignants. Au Brésil, la philosophie n'est pas encore sanctionnée par des examens ni des concours, d'où la nécessité de convaincre l'élève de son intérêt si ce n'est de son utilité pratique.

Ce mouvement interprétatif, qui va de la recherche de représentations à la détermination de causes qui les expliquent, constitue la dernière et la plus interdisciplinaire des étapes d'une recherche en linguistique de discours comparative; ce qui comporte bien sûr des 
risques, puisque les explications causales doivent - comme ce fut le cas pour les marques linguistiques - être cherchées des deux côtés, dans les deux pays étudiés.

\section{BIBLIOGRAPHIE}

BRASIL - SECRETARIA DE EDUCAÇão MÉDIA E TECNOLÓGICA (2000), Parâmetros Curriculares Nacionais para o Ensino Médio. Parte IV Ciências Humanas e suas Tecnologias, Brasília : Ministério da Educação/Secretaria de Educação Média e Tecnológica [en ligne] [site consulté le 20 mars 2013], disponible à l'adresse suivante : http://portal.mec.gov.br/seb/arquivos/pdf/cienciah.pdf BRASIL - SECRETARIA DE EDUCAÇÃO BÁSICA (2006), Orientações curriculares para o ensino médio, volume 3, Brasília : Ministério da Educação/Secretaria de Educação Básica [en ligne] [site consulté le 20 mars 2013], disponible à l'adresse suivante : http://portal.mec.gov.br/seb/ arquivos/pdf/book_volume_03_internet.pdf

BRASIL - MINISTÉRIO DA EDUCAÇÃO, SECRETARIA DE EDUCAÇÃO BÁSICA (2011), Guia de livros didáticos : PNLD 2012 : Filosofia, Brasília.

CARVALHO, Marcelo (2012), «Passé et présent de la philosophie au Brésil » [en ligne], Rue Descartes, 76, traduit par Ana Ferreira Adão [article consulté le 05 mars 2013], disponible à l'adresse suivante : http://www.ruedescartes.org/articles/2012-4-passe-et-present-de-la-philosophie-aubresil/

CHAUI, Marilena (2011), Iniciação à Filosofia, São Paulo : Ática.

COTRIM, Gilberto \& FERNANDES, Mirna (2010), Fundamentos de filosofia, São Paulo : Saraiva.

Delattre, Michel (dir.) (2012), Philosophie. Terminales L, ES, S, Paris : Hatier.

DOISE, Willem \& PALMONARI, Augusto (dir.) (1997), L'étude des représentations sociales, Paris : Delachaux et Niestlé.

GRIZE, Jean-Blaise (1996), Logique naturelle et communications, Paris : Presses Universitaires de France.

HANSEN-LøVE, Laurence (dir.) (2004), Philosophie. Anthologie Terminales L. ES. S, Paris : Belin.

IONESCU, Serban \& BLANCHET, Alain (dir.) (2008), Psychologie sociale et ressources humaines, Paris : Presses Universitaires de France.

конAN, Walter Omar (2007), « La philosophie au Brésil au fil du temps », in Moufida GOUCHA (dir.), La philosophie. Une école de la liberté, Paris : Organisation des Nations Unies pour l'éducation, la science et la culture, 78 .

MARTINS, Maria Helena Pires \& ARANHA, Maria Lúcia de Arruda (2009), Filosofando : introdução à filosofia, São Paulo : Moderna.

MINISTÈRE DE L'ÉDUCATION NATIONALE, « Philosophie au lycée avant la classe terminale » [en ligne], in Site du Ministère de l'Éducation Nationale [page consultée le 31 août 2012], disponible à l'adresse suivante : http://www.education.gouv.fr/cid55161/mene1100064c.html 
MiNISTÈRE DE L'ÉDUCATION NATIONALE, DE L'ENSEIGNEMENT SUPÉRIEUR ET DE LA RECHERCHE. Direction générale de l'enseignement scolaire (2007), Programme Philosophie - classe terminale, série générales et technologiques, Paris : Centre national de documentation pédagogique. Collection textes de référence.

MOREL, Mary-Annick (1996), La concession en français, Paris : Ophrys.

PRESIDÊNCIA DA REPÚBLICA FEDERATIVA DO BRASIL, « Lei 꾼 11.684, de 2 de junho de 2008 » [en ligne], in Site du Planalto brasileiro [page consultée le 01 septembre 2012], disponible à l'adresse suivante : http://www.planalto.gov.br/ccivil_03/_Ato2007-2010/2008/Lei/L11684.htm

RASTIER, François \& PINCEMIN, Bénédicte (1999), « Des genres à l'intertexte », Cahiers de praxématique, 33, 83-111.

RUSS, Jacqueline \& LEGUIL, Clotilde (2012), Les chemins de la pensée. Philosophie Terminales L- ES - S, Paris : Bordas.

SHERRINGHAM, Mark (2006), « L'enseignement scolaire de la philosophie en France » [en ligne], La revue de l'inspection générale, 3 [article consulté le 31 août 2012], disponible à l'adresse suivante : http://media.education.gouv.fr/file/38/6/3386.pdf.

UNESCO (ORGANISATION DES NATIONS UNIES POUR L'ÉDUCATION, LA SCIENCE ET LA CULTURE) (2011),

L'enseignement de la philosophie en Europe et Amérique du Nord, Paris.

VON MÜNCHOW, Patricia (2004), Le journal télévisé en France et en Allemagne. Plaisir de voir ou devoir de s'informer, Paris : Presses Sorbonne-Nouvelle.

VON MÜNCHOW, Patricia (2011), Lorsque l'enfant parait... Le discours des guides parentaux en France et en Allemagne, Toulouse : Presses Universitaires du Mirail.

\section{NOTES}

1. Actuellement, la circulaire $n^{\circ}$ 2011-023 vise à anticiper l'enseignement de la philosophie avant la classe terminale. Cf. http://www.education.gouv.fr/cid55161/mene1100064c.html

2. Presidência da República Federativa do Brasil, « Lei ํํ⒒684, de 2 de junho de 2008 " [en ligne], in Site du Planalto brasileiro [page consultée le $1^{\mathrm{er}}$ septembre 2012], disponible à l'adresse suivante : http://www.planalto.gov.br/ccivil_03/_Ato2007-2010/2008/Lei/L11684.htm

3. Les questions varient seulement en fonction de la voie et de la série dans laquelle l'étudiant est inscrit - voie générale (séries littéraire, économique-sociale ou scientifique) ou technologique étant donné que le nombre d'heures de philosophie n'y est pas le même.

4. D'après le programme officiel du ministère de l'Éducation brésilien, le professeur est libre de choisir le contenu qui sera travaillé en classe (Cf. Brasil - Secretaria de Educação Média e Tecnológica, 2000).

5. Deux heures sont le minimum proposé par le ministère de l'Éducation brésilien pour les cours de philosophie ( $C f$. Brasil - Secretaria de Educação Básica, 2006).

6. Cf. la typologie de Rastier et Pincemin (1999) pour rendre compte de l'organisation des corpus.

7. KANT, E. Critique de la raison pure (1781), partie II, chap. 1, section I, § 3, trad. A. Tremesaygues et B. Pacaud, Éd. des PUF, 1971, p. 505.

8. HEGEL, G. W. F. Leçons sur l'histoire de la philosophie. Introduction du cours de Berlin (1820), trad. J. Gibelin, Éd. Gallimard, coll. «Idées », 1970, pp. 44, 50-51.

9. Idem. Logique, t. I, Germer Ballière, p. 201. Également Précis de l'Encyclopédie des sciences philosophiques, trad. J. Gibelin, Vrin, $5^{\text {e }}$ éd., 1987, p. 39. 
10. C'est nous qui soulignons en gras les passages dans les exemples.

11. Le vestibular est le concours d'entrée à l'université au Brésil.

12. «[...] convaincre c'est "amener quelqu'un, par raisonnement ou par preuves, à reconnaître la vérité ou l'exactitude d'un fait ou sa nécessité". Dans “convaincre" il y a "vaincre". Un argument quasi logique ne s'adresse qu'à la raison [...] Persuader c'est "amener quelqu'un à croire, à faire, à vouloir quelque chose". C'est une intervention qui a pour effet de mobiliser le destinataire et de déclencher en lui une activité qui va entraîner son adhésion totale » (Grize, 1996 : 8-9).

13. «L'ordre dans lequel les notions sont abordées et leur articulation avec l'étude des œuvres relèvent de la liberté philosophique et de la responsabilité du professeur, pourvu que toutes soient examinées " (Cf. Ministère de l'Éducation nationale, de l'Enseignement supérieur et de la Recherche, 2007, p. 6, [c'est nous qui soulignons]).

\section{RÉSUMÉS}

Le thème de cette étude est la comparaison des discours de manuels scolaires de philosophie français et brésiliens. Notre objectif est de dégager les représentations que l'on se fait de la philosophie dans un corpus de manuels français et brésiliens au moyen de l'approche interdisciplinaire qu'est l'analyse du discours ou la «linguistique de discours comparative». Nous exposerons d'abord le contexte de l'enseignement de cette discipline en France et au Brésil. Ensuite, nous présenterons des questions relatives au corpus ainsi qu'au modèle interdisciplinaire adopté pour son analyse, le modèle de description et interprétation (von Münchow, 2004). Quelques hypothèses sur les représentations de la philosophie qui circulent dans les communautés ethnolinguistiques française et brésilienne seront présentées à la fin de cet article.

The theme of this study is the comparison of discourses within French and Brazilian philosophy textbooks. Our purpose is to identify the representations of philosophy existing in a corpus composed of French and Brazilian textbooks through an interdisciplinary approach, that of discourse analysis or "comparative discourse linguistics". First, we will consider the teaching context of this discipline in France and Brazil. Then, we will present some questions relative to the corpus as well as the interdisciplinary model adopted for its analysis, the descriptive and interpretive model (von Münchow, 2004). Some assumptions about the representations of philosophy that circulate in the French and Brazilian ethnolinguistic communities will be presented at the end of this article.

\section{INDEX}

Mots-clés : Analyse du discours, linguistique de discours comparative, manuels de philosophie, France, Brésil

Keywords : Discourse analysis, comparative discourse linguistics, philosophy textbooks, France, Brazil 


\section{AUTEUR}

\section{DANIELA NIENKÖTTER SARDÁ}

\section{Université Paris Descartes}

Laboratoire Éducation et Apprentissages (EA 4071)

Fondation CAPES, Ministère de l'Éducation du Brésil, Brasília - DF 70040-020, Brésil (Boursière CAPES - Proc. no BEX 0911/12-7)

danielasarda@gmail.com 